\title{
The basis of low grain yield and infertility in autotetraploid barley (Hordeum vulgare)
}

\author{
G. M. Evans* and \\ M. M. Rahman $\dagger$
}

\author{
* Department of Agricultural Sciences, \\ University College of Wales, Aberystwyth, \\ Dyfed SY23 3DD, U.K. \\ $\uparrow$ Department of Genetics and Plant Breeding, \\ Bangladesh Agricultural University, Mymensingh, \\ Bangladesh.
}

Under spaced plant conditions the grain yield of autotetraploid forms of two barley cultivars were shown to be less than half that of their diploid counterparts despite an increase in grain size. Lower yield was due to fewer tillers, fewer florets per spike and lower floret fertility. Nevertheless, it is concluded that incomplete seed set remains the main constraint to the utilization of tetraploid barley.

The basis of infertility was examined at both pre and post fertilization stages. Genetic imbalance in aneuploid gametophytes, although a contributory factor, was ruled out as the sole cause of infertility. Non-fertilization of egg cell or polar nuclei and abortion of embryo and/or endosperm during early embryogenesis were also identified as contributing to loss of fertility.

\section{INTRODUCTION}

Most pedigree selection schemes utilise variability generated by hybridization of suitable genotypes. This is so in barley (Hordeum vulgare), a self pollinating diploid having 14 chromosomes. These breeding schemes involve the reassortment and selection of alleles of desirable effects from those already present in cultivated forms of the species. From time to time novel variation is introduced from related species or is generated by mutagen treatment. Induced autotetraploid germplasm also holds some attraction to the breeders on account of their larger seed but the use of such material in barley breeding has been precluded by the lower grain yields associated with such forms (Rommel, 1961; Ulonska and Baumer, 1975; Friedt, 1978). Total yield of grain is the product of the number of flowering heads per plant $\times$ number of seeds per head $\times$ seed size (measured as 100 or 1000 grain weight). The number of seed per head in diploid two-rowed barley is normally described as the number of median florets per head as most if not all these are fertile. However in autotetraploids this is not so and floret fertility assumes a much greater significance. In fact the number of seed per head now becomes the product of florets per head $\times$ per cent floret fertility. The degree of infer- tility of autotetraploids can be quite different depending, to a certain extent, on the species.

The investigation described here had two main objectives. First, it was to determine the extent of the reduction in grain yield of autotetraploid barley compared with diploid counterparts and also to establish by means of a detailed analysis of the components of yield whether factors other than floret fertility were important. Secondly, an attempt was made to determine some of the possible causes of lower fertility in autotetraploid barley. Meiotic chromosome behaviour, pollen grain fertility, pollen tube growth and early embryonic development were all examined in some detail as possible factors which could affect floret fertility as measured at maturity.

\section{MATERIALS AND METHODS}

$\mathrm{C}_{3}$ seeds of four autotetraploid spring barley lines developed from the cultivars Zephyr, Amsel, Balder and Ceresia were obtained from The Welsh Plant Breeding Station, Aberystwyth together with the diploid counterparts of Zephyr and Amsel. All cultivars were multiplied through one more generation to obtain seed of equivalent nutritional status. The tetraploid stocks were screened for 
chromosome numbers and seed taken from eutetraploid plants only.

\section{Field trial}

Diploid and tetraploid Zephyr and Amsel were used for the main trial. Since the tetraploid lines of Balder and Ceresia were available for some of the cytological work described below they were also included here in order to establish their overall yield and components of yield. However, they are not included in the initial analysis on account of there being no diploid equivalents available.

The trial was laid out in a randomised block design with five replicates. A plot comprised of a single row of 12 plants with $20 \mathrm{~cm}$ between plants. The distance between rows was $30 \mathrm{~cm}$. A guard row of a diploid cultivar was planted at each end of every replicate (block) and the end plants of each row were discarded at harvest. Data on plant height, days to flowering, infloresence bearing tillers (spikes/plant), grain yield and harvest index were taken on all ten plants per plot. In addition, the number of florets and of grain per spike and 100 -grain weight were determined on a random sample of five plants from each plot.

\section{Meiosis, pollen development, pollen tube growth and seed development}

\section{Plant culture}

Seed of both diploid and tetraploid stocks were germinated on moist filter paper for five days and then planted in John Innes compost in small pots in an unheated greenhouse from which they were transplanted into the field or into larger pots at a later date.

\section{Meiosis}

Tetraploid stocks of Zephyr, Amsel, Balder and Ceresia, maintained throughout in an unheated greenhouse, were used for this purpose. Diploid Zephyr and Amsel were also included as a check. Inflorescences were fixed and stored in Carnoy's solution until required for examination. Squash preparations of pollen mother cells (PMCs) were prepared in the normal way using aceto-carmine stain. The frequency of the various chromosome configurations and of chiasmata in cells at first metaphase together with the number of chromosomes in anaphase- 1 plates were recorded in a number of plants of each genotype. Since the plants were maintained in the greenhouse floret fertiity was also recorded in these plants at matsiity.

\section{Staining and dissecting of ovule and embryo sac}

Field grown diploid and eutetraploid plants of cultivar Zephyr were used for this purpose. The middle three florets on each side of spikes collected at two, three, four and five days after pollination (anthesis) were fixed in ethanol-acetic acid $(3: 1)$ and stored at room temperature. Ovules were dissected under a low powered microscope in a drop of 45 per cent acetic acid from ovaries which had been stained overnight in Snow's carmine (Snow, 1963). While keeping the ovule in a dorsi-ventral position, a small incision was made at the swollen, i.e., the chalazal end of the ovule using a fine needle. The ovule tissue was gently split apart leaving the embryo sac on the slide. In many instances the embryo at the micropylar end of the embryo-sac could be separated from the endosperm at this stage. In all cases a drop of acetocarmine was added and a coverslip placed in position. Application of gentle pressure to the coverslip was usually necessary to obtain countable nuclei. Slides were examined under a microscope and the various nuclei identified and counted.

\section{Pollen viability, germination and tube growth}

Field grown plants of diploid and tetraploid Zephyr were again used. Mature spikes, just prior to anthesis, were collected, fixed in Carnoy's solution and stored at room temperature in the fixative. Assessment of pollen viability was based on the staining procedure of Alexander (1969). One anther from a median floret was placed in a drop of mixed differential stain, gently macerated, covered with a cover-slip and heated gently over a low flame. Viable (non-aborted) grains appeared deep red while inviable grains were green or partly so, often with shrunken red protoplasm.

Pollen tube development was also assessed on several spikes of both the diploid and tetraploid. Spikes were collected 24 hours after anthesis of the middle spikelets and the middle third of the head fixed in ethanol-acetic acid. Ovaries were carefully dissected and softened in $4 \mathrm{~N} \mathrm{NaOH}$ for three hours at room temperature followed by three washes in distilled water and staining in $0 \cdot 1$ per cent water soluble analine blue in $0 \cdot 1 \mathrm{~N} \mathrm{~K}_{3} \mathrm{PO}_{4}$. Slides were observed under a Zeiss fluorescent microscope using UV light. Fluorescent sieve tubes and pollen tubes were distinguished according to Martin (1959), Kho and Baer (1968) and Chou and Harberd (1970). 


\section{RESULTS}

\section{Field Trial}

The mean values per plant averaged over five replicates for the different characters measured are given in table 1 and a summary of a series of $2 \times 2$ factorial analyses involving diploid and tetraploid Zephyr and Amsel given in table 2. As mentioned earlier, tetraploid Balder and Ceresia were not included in this comparison. This trial confirms once more that autotetraploid forms of $H$. vulgare yield substantially less than their corresponding diploid genotypes. It is also evident, as was expected, that floret fertility was significantly lower in the tetraploids, the difference being 20-24 per cent. However, not only was the lower number of grains per spike in the tetraploids due to lower fertility, but it was also partly due to a significantly lower number of florets per spike. The yield potential of the tetraploids was further reduced by these forms producing significantly fewer spike bearing tillers under this spaced plant growing regime. Extrapolation of this data gives an overall reduction of 64 per cent in the number of grains per plant in the tetraploids when compared with the diploids. In contrast to these deleterious effects of change in ploidy, the individual grain size as measured by 100 -grain weight is significantly larger in the tetraploids, the difference being in the region of 18 per cent. Although the tetraploids were in -general shorter than their diploid counterparts their harvest index was substantially and significantly lower. It should be noted that harvest index of all genotypes is on the low side on account of the inclusion of the root ball in the total weight of plants.

Highly significant differences in days to flower, number of spikes per plant and 100-grain weight between the two cultivars is of no particular interest in this investigation. Of greater interest is the cultivar $\times$ ploidy interaction component in table 2 .
That the interaction item is very low for all three parameters indicates that the relative effect of tetraploidy is similar in both cultivars. The one possible exception is that of harvest index where the interaction item is marginally significant $(P=0.05)$. This is due to Zephyr having a higher harvest index at the diploid level but not at the tetraploid level.

It is worth noting at this stage that the results obtained for the tetraploid versions of Balder and Ceresia are on the whole similar to those obtained for tetraploid Zephyr and Amsel. The number of grains per spike is again reduced by low fertility and although the number of florets per head is also low no definitive conclusions can be made in the absence of any diploid counterparts.

\section{Meiosis}

Data on chromosome association at metaphase-1 and of the separation at anaphase- 1 of meiosis are given in table 3. Also included is the floret fertility which was estimated from the same plants used for analysis of meiosis. The values are therefore marginally different from those obtained from the field trial and given in table 1. The term "efficient PMC" was based on that used by Hazerika and Rees (1967) and in this context denotes pollen mother cells which do not contain trivalents or univalents.

The mean values for all the characters measured are very similar for all the four cultivars although there is considerable plant to plant variation within each variety. The number of quadrivalents per cell is in line with that expected in an autotetraploid with a chiasma frequency of 24-25. There were relatively few trivalents and univalents and this is reflected in the high values for "per cent efficient PMC". Since the objective of this experiment was to determine whether floret fertility could be related to any facet of meiosis a series of correlations were carried out on the individual plant data

Table 1 Mean plant values for a series of agronomic, yield and yield component characters of two diploid and four tetraploid lines of barley

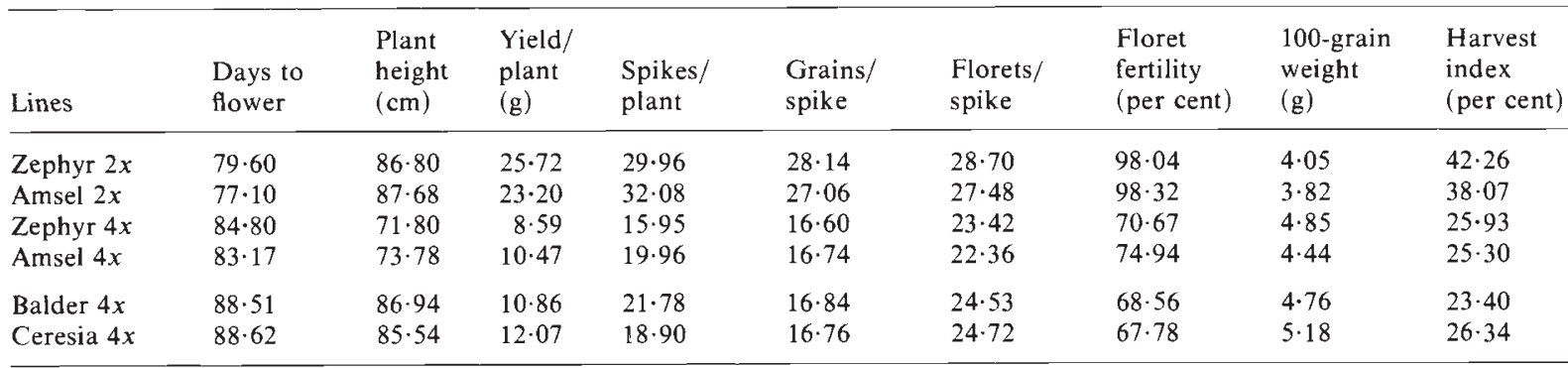




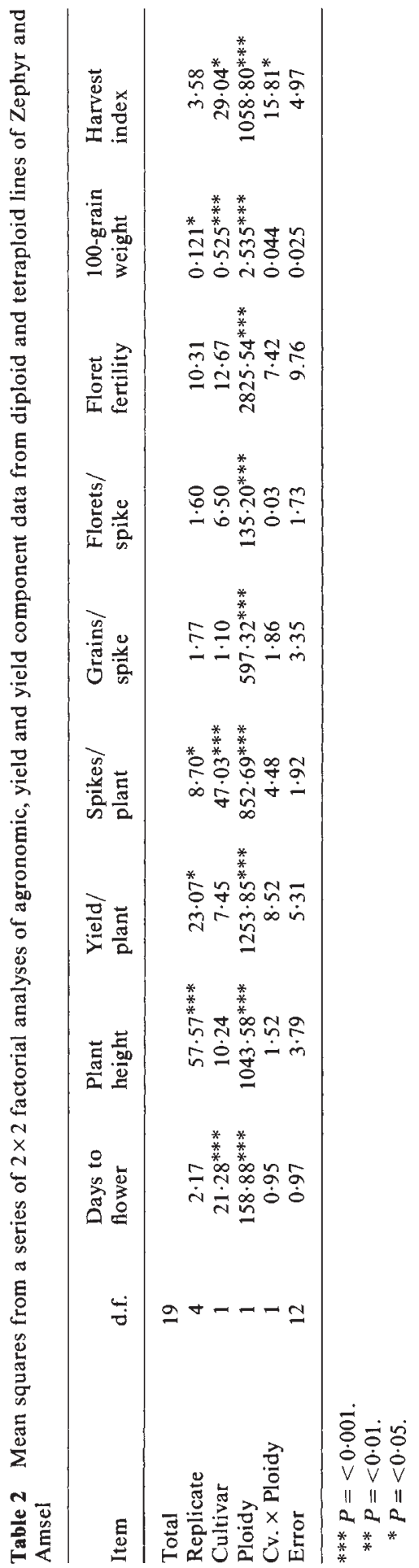


Table 3 Mean frequencies and range (brackets) of the different metaphase-1 configurations and of chiasmata together with the percentage of efficient PMCs, percentage of anaphase-1 14 chromosome complements and percentage of floret fertility in autotetraploid lines of barley

\begin{tabular}{|c|c|c|c|c|c|c|c|c|c|}
\hline \multirow[b]{2}{*}{ Lines } & \multirow[b]{2}{*}{$N$} & \multicolumn{5}{|c|}{ Frequency per pollen mother cell of } & \multirow{2}{*}{$\begin{array}{l}\text { Efficient } \\
\text { PMC } \\
\text { (per cent) }\end{array}$} & \multirow{2}{*}{$\begin{array}{l}14 \text { 's at } \\
\text { An.1 } \\
\text { (per cent) }\end{array}$} & \multirow{2}{*}{$\begin{array}{l}\text { Floret } \\
\text { fertility } \\
\text { (per cent) }\end{array}$} \\
\hline & & IV & III & II & I & Chiasmata & & & \\
\hline Zephyr & 5 & $\begin{array}{l}3 \cdot 22 \\
(2 \cdot 6-3 \cdot 7)\end{array}$ & $\begin{array}{l}0 \cdot 08 \\
(0-0 \cdot 2)\end{array}$ & $\begin{array}{l}7 \cdot 38 \\
(6 \cdot 4-8 \cdot 5)\end{array}$ & $\begin{array}{l}0 \cdot 12 \\
0-0 \cdot 3)\end{array}$ & $\begin{array}{l}25 \cdot 56 \\
(24 \cdot 4-26 \cdot 5)\end{array}$ & $\begin{array}{l}90 \cdot 0 \\
(80 \cdot 0-100 \cdot 0)\end{array}$ & $\begin{array}{l}72 \cdot 5 \\
(52 \cdot 5-87 \cdot 5)\end{array}$ & $\begin{array}{l}69 \cdot 69 \\
(61 \cdot 1-82 \cdot 6)\end{array}$ \\
\hline Amsel & 4 & $\begin{array}{l}3 \cdot 05 \\
(2 \cdot 7-3 \cdot 3)\end{array}$ & $\begin{array}{l}0 \cdot 13 \\
(0-0 \cdot 4)\end{array}$ & $\begin{array}{l}7 \cdot 65 \\
(7 \cdot 2-8 \cdot 6)\end{array}$ & $\begin{array}{l}0 \cdot 12 \\
(0-0 \cdot 4)\end{array}$ & $\begin{array}{l}24 \cdot 83 \\
(24 \cdot 5-26 \cdot 1)\end{array}$ & $\begin{array}{l}92 \cdot 5 \\
(80 \cdot 0-100 \cdot 0)\end{array}$ & $\begin{array}{l}76 \cdot 9 \\
(60 \cdot 0-92 \cdot 5)\end{array}$ & $\begin{array}{l}74 \cdot 88 \\
(68 \cdot 3-84 \cdot 1)\end{array}$ \\
\hline Balder & 4 & $\begin{array}{l}2 \cdot 57 \\
(2 \cdot 0-2 \cdot 8)\end{array}$ & $\begin{array}{l}0 \cdot 08 \\
(0-0 \cdot 2)\end{array}$ & $\begin{array}{l}8 \cdot 65 \\
(7 \cdot 4-9 \cdot 8)\end{array}$ & $\begin{array}{l}0 \cdot 18 \\
(0-0 \cdot 4)\end{array}$ & $\begin{array}{l}24 \cdot 73 \\
(24 \cdot 5-25 \cdot 3)\end{array}$ & $\begin{array}{l}87 \cdot 5 \\
(80 \cdot 0-100 \cdot 0)\end{array}$ & $\begin{array}{l}71 \cdot 9 \\
(65 \cdot 0-85 \cdot 0)\end{array}$ & $\begin{array}{l}66 \cdot 26 \\
(62 \cdot 86-70 \cdot 42)\end{array}$ \\
\hline Ceresia & 5 & $\begin{array}{l}3 \cdot 02 \\
(2 \cdot 7-3 \cdot 5)\end{array}$ & $\begin{array}{l}0 \cdot 18 \\
(0 \cdot 1-0 \cdot 4)\end{array}$ & $\begin{array}{l}7 \cdot 58 \\
(6 \cdot 8-8 \cdot 0)\end{array}$ & $\begin{array}{l}0 \cdot 22 \\
(0 \cdot 1-0 \cdot 4)\end{array}$ & $\begin{array}{l}25 \cdot 32 \\
(24 \cdot 5-26 \cdot 1)\end{array}$ & $\begin{array}{l}80 \cdot 0 \\
(60 \cdot 0-90 \cdot 0)\end{array}$ & $\begin{array}{l}62 \cdot 5 \\
(55 \cdot 0-67 \cdot 5)\end{array}$ & $\begin{array}{l}72 \cdot 67 \\
(60 \cdot 26-80 \cdot 60)\end{array}$ \\
\hline Mean & & $2 \cdot 97$ & $0 \cdot 12$ & $7 \cdot 82$ & $0 \cdot 16$ & $25 \cdot 11$ & $87 \cdot 5$ & $70 \cdot 94$ & $71 \cdot 13$ \\
\hline
\end{tabular}

with all four varieties combined. These are given in table 4. There is no indication whatsoever of a correlation between any of the meiotic parameters and fertility. The data does show however that PMCs which do not contain trivalents or univalents ("efficient PMCs") tend to give disjunctional separation of chromosomes at anaphase-1 although the correlation of per cent efficient PMCs and 14 chromosome anaphase- 1 complements is not as strong as might be expected. This would imply that some quadrivalents occasionally do not separate $2: 2$ and/or that trivalents and univalents separate equationally at anaphase-1. It is likely to be the former since the proportion of PMCs showing equational (14:14) separation at anaphase-1 is much lower than the per cent efficient PMCs at metaphase. This apparently has no effect on fertility. It has to be borne in mind that the meiotic analysis is done solely on pollen mother cells and the assumption is made that the pattern of meiosis is similar in the embryo sac mother cells. This will be discussed later.

A check on meiosis in diploid Zephyr and Balder showed a completely normal process with invariably seven bivalents at metaphase-1 and 7-7 separation at anaphase-1. The data is not shown here.

\section{Early seed development}

The development of the ovary, ovule and embryo sac content from diploid and tetraploid Zephyr were closely examined on days two to five after pollination (anthesis). It was clear at the outset that the ovaries could be separated into two distinct types based on their shape. The "normal" development involved a gradual elongation from $3 \mathrm{~mm}$ to $7 \mathrm{~mm}$ by day 5 with only a small increase in ovary breadth. On the other hand a substantial number of ovaries from the tetraploid line showed "abnor-

Table 4 Correlation coefficient values between meiotic characters and floret fertility in the four tetraploid lines of barley

\begin{tabular}{lcr}
\hline & $\begin{array}{l}\text { PMCs with 14 } \\
\text { chromosomes at } \\
\text { anaphase-1 } \\
\text { (per cent) }\end{array}$ & $\begin{array}{l}\text { Floret } \\
\text { fertility } \\
\text { (per cent) }\end{array}$ \\
\hline Frequency of quadrivalents & 0.19 & 0.16 \\
Frequency of trivalents & -0.42 & 0.01 \\
Frequency of bivalents & -0.05 & -0.15 \\
Frequency of univalents & $-0.55^{*}$ & -0.13 \\
Frequency of chiasmata & 0.21 & 0.22 \\
Per cent efficient PMCs & $0.58^{*}$ & -0.03 \\
Per cent 14 chrom. A1 cells & & -0.05 \\
\hline
\end{tabular}

$* P=0 \cdot 05 ; N=18$ 
Table 5 Percentage normal ovaries at days 2-5 after pollination and of seed set at maturity in diploid and tetraploid Zephyr barley

\begin{tabular}{lll}
\hline & \multicolumn{2}{l}{ Florets with normal ovaries/seed in } \\
\cline { 2 - 3 } $\begin{array}{l}\text { Days after } \\
\text { pollination }\end{array}$ & $\begin{array}{l}\text { Diploids } \\
\text { (per cent) }\end{array}$ & $\begin{array}{l}\text { Tetraploids } \\
\text { (per cent) }\end{array}$ \\
\hline 2 & $100 \cdot 00$ & $64 \cdot 71$ \\
3 & $100 \cdot 00$ & $70 \cdot 37$ \\
4 & 100.00 & 62.00 \\
5 & 95.24 & 66.67 \\
Maturity & 95.49 & 69.69 \\
\hline
\end{tabular}

mal" development. These showed some lateral swelling but no elongation. As might be expected from this type of development the ovules in abnormal ovaries were short and did not extend beyond the narrow base while those showing normal development extended almost to the tip of the ovary. The percentage florets with normal ovaries on days 2-5 for both the diploid and tetraploid is given in table 5 . Nearly all the diploid ovaries were of the "normal" type with only one out of 21 ovaries examined at day 5 being abnormal. On the other hand the number of normal ovaries in the tetraploid was significantly lower with the values ranging from 62 to 70.37 per cent. However the values do not show any particular trend over these four days of early development. Moreover the proportion of ovaries which appear normal is very close to that of seed set at maturity recorded in this experiment (table 5) and to the 70.67 per cent obtained in the field trial (table 1). This would indicate that there was either failure of fertilization or of embryonic breakdown at a very early stage in the florets which did not set seed.

A detailed description of the development of embryo and endosperm is not given here. Suffice to comment that by day 5 the embryos of the tetraploids had gone through 7.58 and the endosperm 13.86 cell cycles. Comparable figures for the diploids were 7.98 and 14.60 for embryo and endosperm. These were calculated from counts of nuclei/cells and using the formula:

$$
\text { Number of cell cycles }=\frac{\log x}{\log 2},
$$

where $x$ denotes the number of embryo cells or endosperm nuclei (cells).

Of greater interest in terms of loss of fertility was the contents of ovules from the abnormal ovaries from the tetraploid line. Ovules from 61 abnormal ovaries were dissected over the four days with 11-21 analysed per day. Data of contents are given in table 6. Apart from the endoreplicated antipodal cells it was impossible to identify any other contents in a proportion of the ovules examined and this proportion increased with time (from 43.8 per cent on day 2 to 90.5 per cent on day 5). The contents of those which could be analysed showed that cessation of development was accompanied by a range of abnormality of ovule content. Unfertilized egg cells were identified in three ovules and a pair of haploid polar nuclei which, rather suprisingly, appeared to be at late mitotic prophase was clearly visible in one. Ovules with degenerating embryos and/or endosperm were quite common at days 2 and 3 . Six out of 16 ovules examined on day 2 and five out of 11 on day 3 contained either degenerating darkly stained embryos and/or endosperm. While some degenerating ovaries were also identified at days 4 and 5, no endosperm was present at this stage. Cell counts of degenerating embryos and endosperm showed that the embryos, in particular, had undergone several cycles of nuclear divisions. The maximum for the samples analysed was five on day 4 and the average for all the degenerating embryos on this day was $4 \cdot 61$. That for normal

Table 6. Frequency of ovules with egg cell, polar nuclei, embryo and endosperm in abnormal ovaries

\begin{tabular}{|c|c|c|c|c|}
\hline & \multicolumn{4}{|c|}{ Days after pollination } \\
\hline & 2 & 3 & 4 & 5 \\
\hline Ovules examined & 16 & 11 & 13 & 21 \\
\hline Ovules with egg cell & 2 & 0 & 1 & 0 \\
\hline Ovules with unfertilized polar nuclei & 1 & 0 & 0 & 0 \\
\hline Ovules with degenerating embryo & 3 & 4 & 2 & 2 \\
\hline Ovules with degenerating endosperm & 4 & 3 & 0 & 0 \\
\hline (Ovules with both embryo and endosperm & 1 & 2 & 0 & $0)$ \\
\hline Ovules with no identifiable content & 7 & 6 & 10 & 19 \\
\hline (\% ovules with no identifiable content & $43 \cdot 8$ & $54 \cdot 5$ & 76.9 & $90 \cdot 5)$ \\
\hline
\end{tabular}


embryos was $5 \cdot 71$. Bearing in mind that normal endosperm divides at a much faster rate than embryos, it appeared that the endosperm ceased to develop at an earlier stage. All three degenerating embryos identified on day 3 had undergone four cell cycles whereas the normal endosperm had undergone, on average, 11.5 cell cycles. The number and behaviour of antipodal cells was also different in the abnormal ovaries. In both the diploid and normal tetraploid catagories the number of these decreased from a maximum of around 50 on day 2 to almost complete degeneration by day 5 . In the abnormal ovaries they were fewer in number at around 30 cells and they remained constant in number throughout the sampling period.

\section{Pollen viability, germination and tube growth}

Pollen variability as determined by the staining method was, as expected, very high in the diploid with a mean value of 98.19 per cent from 20 anthers. Comparable values for the tetraploid were $82 \cdot 19$ per cent from 48 anthers. Clearly there was no shortage of viable pollen in the anthers of tetraploid plants. This was confirmed by an assessment of pollen germination on the stigmatic surface one day after anthesis. Pollen germination on all stigmatic surfaces was extensive and pollen tubes were detected in all stylar and ovarian tissues. Although there were a number of short pollen tubes which had not penetrated the stigmatic surface, no difference in number could be detected between stigmas of different ovaries some of which would be of the abnormal type. More than one pollen tube was detected in all ovarian tissues of both the diploid and tetraploid line. No attempt was made to quantify this nor was it possible to determine whether the discharge of sperm nuclei was normal in all tetraploid florets.

\section{DISCUSSION}

The inevitable conclusion from the grain yield comparisons is that the diploids are vastly superior to their tetraploid counterparts and indeed the tetraploids as a group. As single plants the yield of the tetraploids was less than half that of the diploids despite having slightly larger seed.

Lower grain yields in tetraploid barley has been reported previously by Rommel (1961), Ulonska and Baumer (1975) and by Friedt $(1978,1985)$ and the present results are in line with these. Similarly, seed yield of rye is also reduced by tetraploidy (Muntzing, 1951; Aastveit, 1968; Pfahler, 1987) but apparently not as drastically as in barley
(Friedt, 1985). On the other hand, the seed yields of tetraploid forage crops such as the Loliums and Trifolium pratense can be higher than the diploids (Julen, 1975; van Bogaert, 1975).

Two possible reasons for this different response to polyploidy are apparent. First, the increase in seed size associated with increased ploidy is much larger in nongrain crops, i.e., crops which have not been subjected to selection for seed size during their domestication and evolution. In the forages such as the ryegrasses and red clover, the lower fertility of the tetraploids is more than offset by the large increase in grain size. Whereas the increase in 100-seed weight of the two tetraploid barley varieties in the present experiment was only 18 per cent and that recorded by Pfahler (1987) for rye was an average 16 per cent, the increase in seed size of $L$. perenne and $L$. multiflorum was estimated by van Bogaert (1975) as 82.9 per cent and 84.2 per cent respectively. Secondly, it has been suggested (Friedt, 1985) that outbreeders as a group are more tolerant of increased ploidy than are inbreeders although the evidence for this is largely circumstantial.

The basis of this sharp decrease in yield of the tetraploids is the deleterious effect of polyploidy on a range of plant characteristics which ultimately determine the yielding capacity of a grain crop. The fertility, tillering capacity and the number of florets per head were all drastically reduced. An improvement in these three parameters is therefore essential before the benefits of larger grain size can be realised. Reduction in tillering capacity probably does not present such a major problem as it should be feasible to manipulate the seeding rate to obtain the necessary number of tillers per hectare of land. It should also be borne in mind that the difference in tillering capacity between diploids and tetraploids is unlikely to be as extensive under normal culture regimes. Although improvement in the number of florets per spike presents a more difficult problem some progress should be possible through breeding. Extensive variation is known to exist amongst diploid cultivars for this character (Riggs et al., 1981).

Loss of fertility remains therefore the major constraint to the use of tetraploid forms of barley. Kao, Reinbergs and Harvey (1970) and Friedt (1985) have shown that small improvements in overall fertility of tetraploid barley can be achieved by hybridization and selection over several generations. However, the highest levels of 77 per cent and 76 per cent fertility recorded in these two programmes fall far short of that required for a grain crop. 
The idea that meiotic irregularities are responsible for low fertility through the production of genetically imbalanced products is widely held and is based on correlations between the pattern of chromosome pairing and/or disjunction at meiosis and floret fertility at maturity in the same populations. These include Hilpert (1957), Hazerika and Rees (1967) and Aastveit (1968) in rye, Crowley and Rees (1968) in ryegrass, Jahuar (1970), Narasinga Rao and Pantulu (1982) and Arundhati, Pantulu and Narasinga Rao (1983) in pearl millet and Kao, Reinbergs and Harvey (1969), Reinbergs, Kao, Harvey and Shebeski (1970) and Fedak (1975) in barley. However, the evidence is far from conclusive and at times it is even conflicting. For example, whereas Hazerika and Rees (1967) reported a positive correlation between quadrivalent frequency and fertility, Moore (1963) found no correlation between these traits in different populations of tetraploid rye. Conflicting evidence in other species follows the same pattern. Reinbergs et al. (1970) and Fedak (1975) found a correlation between distribution of chromosomes at anaphase1 of meiosis and fertility in tetraploid barley whereas no such correlation was apparent in the present analysis. Indeed we found no correlation between any meiotic parameter and fertility.

A knowledge of the origin and fate of aneuploid gametophytes is of relevance to the interpretation of the type of analysis reported above. Aneuploid products are the consequence of non-disjunction of chromosomes at first anaphase of meiosis. Although this is primarily due to the presence of trivalents and univalents at earlier stages it can also be due to unequal separation of certain types of quadrivalents. There is no doubt that some aneuploid gametes are inviable and contribute to infertility but it is also abundantly clear that a large proportion of such products are nevertheless viable, hence the occurrence of a substantial number of aneuploid individuals in most autotetraploid populations. However, the majority of these would differ from the euploid complement by only one chromosome. Although this is in part due to the fact that a large proportion of the aneuploid products of meiosis fall into this class, it is also likely that gametophytes which deviate from the euploid number by several chromosomes do not survive. The likelihood of these occurring increases with a decrease in chiasma frequency of meiocytes because of the increased frequency of univalents and trivalents. It also follows that the greater the variation in chiasma frequencies and therefore of trivalent and univalent frequency the higher the probability of obtaining a significant correlation between these parameters and fertility. That some workers have found such correlations while others have not is therefore probably due to the different degree of variability of the populations being examined. In the present study the range of chiasma frequencies was low and it is not surprising therefore that no correlations with fertility were found. Even in the absence of such correlations the presence of aneuploid gametes could still contribute to infertility but it is unlikely to be the only factor in the present material. Although the frequency of euploid anaphase complements were in the same order as that of fertility (table 3 ), it has to be borne in mind that many of the aneuploid anaphase complements would form viable gametes. The frequency of aneuploids in these populations appears to be in the region of $25-30$ per cent (Rahman, 1985).

The basic assumption that the pattern of chromosome association and separation is the same in embryo sac mother cells and pollen mother cells is untested in most species, especially at the tetraploid level. All the analyses reported above use pollen mother cell data with the full knowledge that it is the viability of the embryo sac and its derivatives which determine the level of fertility in most cases. Rahman (1985) showed that at least in a small sample of EMCs from tetraploid $L$. temulentum $\times L$. perenne the overall pattern of metaphase 1 was similar to that in PMCs. It does not follow that this is so in all species and even in all genotypes within species. Small differences in the frequency and distribution of chiasmata could make a substantial difference to the frequency of the various configurations at metaphase in a tetraploid.

No detail of pre-fertilization events in the embryo sac were obtained from this present study. However, it was clear that ovaries which were phenotypically abnormal could be detected as early as two days after pollination. These were clearly developmentally abnormal as well. They did not develop to maturity even though some of them contained embryo and endosperm cells. Since the relative frequency of these did not change significantly between day 2 and day 5 , it could be concluded that some abnormality had occurred prior to day 2 and this was irreversible. The ovaries which had a normal phenotype developed to maturity and there was very little difference, apart from the number of cells, in embryos and endosperm from these and from full fertile diploid florets.

It is reasonable to suggest that failure of normal embryo sac development could account for some 
abnormal ovaries at a later date. This could be the consequence of gross aneuploidy or of some other form genetic imbalance due to tetrasomy. Embryo sacs with no apparent content, as seen on day 2 of the present study, could be the consequence.

Pollination and the subsequent growth of pollen tubes appeared to be adequate in the tetraploid, although failure of discharge of sperm nuclei into the embryo sac cannot be ruled out. Indeed at day 2 there were a small proportion of ovules with unfertilized egg cell or polar nuclei. Abortion of embryo and endosperm during the early stages of development also accounted for some loss of seed set. Again there is no ready explanation for this as the nuclei had undergone as many as five cell cycles in some cases. Bennett, Smith and Barclay (1975) suggest that the antipodals are concerned with supplying the protein requirements of the rapidly dividing coenocytic endosperm. The number and behaviour of these cells was certainly different in the abnormal ovaries. Not only were they fewer in number they also did not degenerate during early embryognesis. This still does not distinguish between the cause and effect of degenerating endosperm.

In conclusion therefore, it appears that abnormality in ovarian growth resulting in "sterile" florets in mature plants of autotetraploid barley is due to several causes. These include failure of fertilization of the egg or polar nuclei, degeneration of the embryo and/or endosperm during early embryogenesis and probably the abnormal development of the embryo sac prior to pollination.

\section{REFERENCES}

AASTVEIT, K. 1968. Variation and selection for seed set in tetraploid rye. Hereditas, 60 , 294-316.

ALEXANDER, M. P. 1969. Differential staining of aborted and non-aborted pollen. Stain Technol., 44, 117-122.

ARUNDHATI, K., PANTULU, J. V. AND NARASINGA RAO, P. S. R. L. 1983. Effect of selection for seed set on meiotic chromosome behaviour in autotetraploid pearl millet. $Z$. Pflanzenzuchtung, 90, 145-152.

BENNETT, M. D., SMITH, J. B. AND BARCLAY, I. 1975. Early seed development in the Triticeae. Phil. Trans. R. Soc. Lond. $B, 272,199-227$.

CHOU, M. C. AND HARBERD, D. J. 1970. Note on visual distinction of fluorescent callose of pollen tubes and sieve tubes in stylar tissue of Brassica and its allies. Euphytica, 19, 379-381.

CROWLEY, J. G. AND REES, H. 1968. Fertility and selection in tetraploid Lolium. Chromosoma, 24, 300-308.

FEDAK, G. 1975. Fertility and meiotic behaviour in tetraploid barley. Canad. J. Genet. Cytol., 17, 121-123.
FRIEDT, W. 1978 Untersuchungen an autotetraploiden gersten unter besonderer Berücksichtigung der diploidisierung. I. Frtilitat, vitalitat and kornentrag. Z. Pflanzensuchtung, 81, 118-139.

FRIEDT, W. 1985. The present status of breeding autotetraploid cereals. In Horn, W. and Jensen, C. J. (eds) Genetic Manipulation and Plant Breeding, EUCARPIA, Berlin, pp. 123-128.

HAZERIKA, M. H. AND REES, H. 1967. Genotypic control of chromosome behaviour in rye. $\mathrm{X}$. Chromosome pairing and fertility in autotetraploids. Heredity, 22, 317-332.

HILPERT, G. 1957. Effect of selection for meiotic behaviour in autotetraploid rye. Hereditas, 43, 318-322.

JAHUAR, P. P. 1970. Chromosome behaviour and fertility of raw and evolved synthetic tetraploids of pearl millet, Pennisetum typhides Stapf. Et Hubb. Genetica, 41, 407-424.

JULEN, G. 1975. The current situation in tetraploid clover. In Nuesch, Z. B. (ed.) Ploidy in Fodder Crops, EUCARPIA, Zurich, pp. 79-89.

KAO, K. N., REINBERGS, E. AND HARVEY, B. L. 1969. Fertility and preferential chromosome pairing in autotetraploid barley. Canad. J. Genet. Cytol., 11, 479-480.

KAO, K. N., REINBERGS, E. AND HARVEY, B. L. 1970. Selection for seed setting in hybrid populations of autotetraploid barley Hordeum vulgare L. emend Lam. Crop Sci., 10, 491-492.

KHO, Y. O. AND BAER, J. 1968. Observing pollen tubes by means of fluorescence. Euphytica, 17, 298-303.

MARTIN, F. W. 1959. Staining and observing pollen tubes in the style by means of fluorescence. Stain Technol., 34, $125-128$.

MOORE, K. 1963. The influence of climate on a population of tetraploid spring rye. Hereditas, 49, 269-305.

MUNTZING, A. 1951. Cytogenetic properties and practical value of tetraploid rye. Hereditas, 37, 17-84.

NARASINGA RAO, P. S. R. L. AND PANTULU, J. V. 1982. Fertility and meiotic chromosome behaviour in autotetraploid pearl millet. Theor. Appl. Genet., 62, 345-351.

PFAHLER, P. L., BARNET, R. D. AND LUKE, H. H. 1987. Diploid/tetraploid comparisons in rye. IV. Grain production. Crop Sci., 27, 431-435.

RAHMAN, M. M. 1985. Fertility and Stability of Tetraploid Grasses. Ph.D. Thesis, University of Wales.

REINBERGS, E., KAO, K. N., HARVEY, B. L. AND SHEBESKI, L. H. 1970. Meiotic behaviour and preferential pairing in autotetraploid barley. Crop Sci., 10, 569-571.

RIGGS, T. J., HANSON, P. R., START, N. D., MILES, D. M., MORGAN, C. L. AND FORD, M. A. 1981. Comparison of spring barley varieties grown in England and Wales between 1880 and 1980. J. agric. Sci., Camb., 97, 599-610.

ROMMEL, M. 1961. Aneuploidy, seed set and sterility in artificially induced autotetraploid Hordeum vulgare L. Can. J. Genet. Cytol., 3, 272-282.

SNOW, R. 1963. Alcoholic hydrochloric acid-carmine as a stain for chromosomes in squash preparations. Stain Technol., $38,9-13$.

ULONSKA, E. AND BAUMER, M. 1975. Investigation for the improvement of yield and quality in hull-less and naked autotetraploid barley. In Barley genetics III, Proceedings Third International Genetic Symposium, pp. 388-395.

VAN BOGAERT, G. 1975. A comparison between colchicine induced tetraploid and diploid cultivars of Lolium species. In Nuesch, Z. B. (ed.) Ploidy in Fodder Crops, EUCARPIA, Zurich, pp. 61-68. 